\title{
Effect of Ga Incorporation and Film Thickness on the Optical Properties of as-Deposited Amorphous $\mathrm{Ga}_{\mathbf{x}} \mathrm{Se}_{1-\mathrm{x}}$ Thin Films
}

\author{
Austine A. Mulama ${ }^{1,2 *}$, Julius M. Mwabora ${ }^{1}$, Andrew O. Oduor ${ }^{2}$, Cosmas M. \\ Muiva $^{3}$, and Chrispinus M. Walloga ${ }^{4}$ \\ ${ }^{I}$ Physics Department, University of Nairobi, Kenya \\ ${ }^{2}$ Physics and Materials Science Department, Maseno University, Kenya \\ ${ }^{3}$ Physics Department, Botswana International University of Science and Technology, Botswana \\ ${ }^{4}$ Physics Department, Mount Kenya University, Kenya
}

\begin{abstract}
Flash evaporated amorphous $G a_{x} S e_{1-x}(x=0,1,2,3$, and 4 atomic \%) on glass substrates have been investigated within a 500nm-1500nm spectral range. Film thicknesses explored were ; 200 $110 \mathrm{~nm}, 265 \pm 10 \mathrm{~nm}$, $330 \pm 10 \mathrm{~nm}$, and $400 \pm 10 \mathrm{~nm}$. The effect of film thickness and gallium content on the as-deposited thin films has been established. As the gallium content increases, both the optical transmittance and band gap energy decrease. Increase in film thickness led to a decrease in optical transmittance and an increase in the band gap energy. The other optical parameters like the absorption coefficient, extinction coefficient, refractive index, real part and imaginary part of dielectric constant increase with increase in gallium content and film thickness.
\end{abstract}

Keywords: Amorphous thin film, Film thickness, Ga-Se alloy, Optical property

\section{Introduction}

Selenium is one of the chalcogen elements of group 6 in the periodic table (selenium, sulphur and tellurium) [1]. It is both a photoconductive and a photovoltaic material. As a result, it is useful in the formation of photo cells and solar cells. In addition, it can convert alternating current to direct current electricity, and therefore extensively used in the formation of rectifiers. However, pure amorphous selenium (a-Se) is less photosensitive, thermally unstable and easily crystallizes at room temperature [2]. This has been overcome by alloying a-Se with other elements to make it stable. Several elements including lead (Pb), arsenide (As), bismuth $(\mathrm{Bi})$, indium $(\mathrm{In})$, gallium $(\mathrm{Ga})$, have been used to alloy a-Se matrix $[1,3,4]$. Gallium $(\mathrm{Ga})$ readily alloys well with most metals and semiconductors to yield different alloys for the formation of solid-state devices such as transistors, both in bulk and thin film form. Use of Ga instead of In in copper-indium-diselenide solar cells leads to increase in open circuit voltage and band gap energy [5]. Although studies on a-Ga-Se alloy thin films have been done, effect of film thickness on the optical properties of a-Ga-Se thin films is a rarity. Mustafa et al [6] considered different thicknesses of a-Ga-Se alloy thin films but did not investigate the effect of film thickness on the optical properties of the deposited films. The study therefore investigates the effect of film thickness on the optical properties of as-deposited a-Ga-Se thin films in addition to Ga content.

\section{Materials and methods}

The bulk samples of $\mathrm{Ga}_{\mathrm{x}} \mathrm{Se}_{1-\mathrm{x}}(\mathrm{x}=0,1,2,3$, and 4 atomic \%) were prepared using the well known melt quenching method. Pure (99.99\%) gallium and selenium were weighed according to given percentages. The weighed samples were sealed in evacuated quartz ampoules $\left(4.0 \times 10^{-5} \mathrm{mbar}\right)$ and heated in a rotatable programmable furnace to $500^{\circ} \mathrm{C}$ at a rate of $3^{\circ} \mathrm{C}$ per minute. Chalcogenide glasses belong to substances which have an incongruent melting point, exhibit a high partial vapour pressure during melting and the melt tend to have a high viscosity and are susceptible to oxidation and hydrolysis. Therefore, the synthesis must be carried out in sealed evacuated quartz ampoules $[1,4,7]$. The heating was maintained at $500^{\circ} \mathrm{C}$ for 12 hours to get a uniform mixture of the samples. The quartz ampoules were quenched in ice-cooled water. Quenching ensures that the different atoms are randomly mixed to form continuous homogeneous non-crystalline films on cleaned substrates, the films having greater than stoichiometric proportions of the non-metal component. The glass forming region obtained by quenching in ice water is much greater than that obtained from quenching in air or slow cooling. The quartz ampoules were then broken and the mixture crushed for flash evaporation (Fig. 1). The vibrator was used for dispensing powder onto the boat at a direct current voltage of $15 \mathrm{~V}$. The vibrations were transmitted to the silica tube. The powder then fell grain by grain from the vibrating material tube into the silica tube to the preheated boat below it. The substrate-source distance was $11 \mathrm{~cm}$ to ensure complete deposition of the alloy on the corning glass substrates. X-Ray patterns were measured on XRD machine, PW3710, UK. Film thicknesses were measured on a surface profiler with a resolution of $10 \mathrm{~nm}$. The transmittance and reflectance were measured on Solidspec 3700 DUV, Kyoto-Japan (500nm-1500nm). 


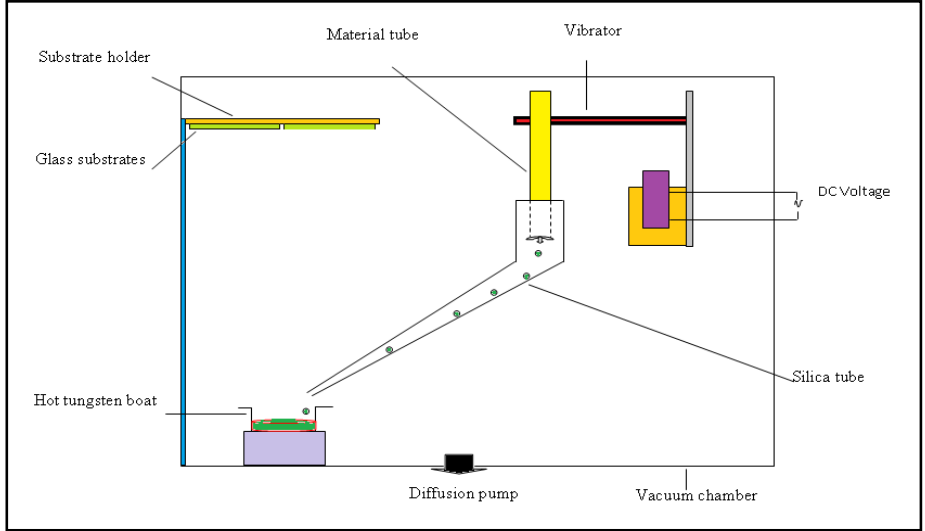

Figure 1: Setup for flash evaporation method

\section{Results And Discussions}

\subsection{Nature of as-deposited thin films}

Measured film thicknesses were: $200 \pm 10 \mathrm{~nm}, 265 \pm 10 \mathrm{~nm}, 330 \pm 10 \mathrm{~nm}$, and $400 \pm 10 \mathrm{~nm}$. The X-Ray diffraction patterns shown in Fig. 2 indicate that the as-deposited thin films are amorphous due to absence of sharp peaks. The humps are as a result of the glass substrate [8].

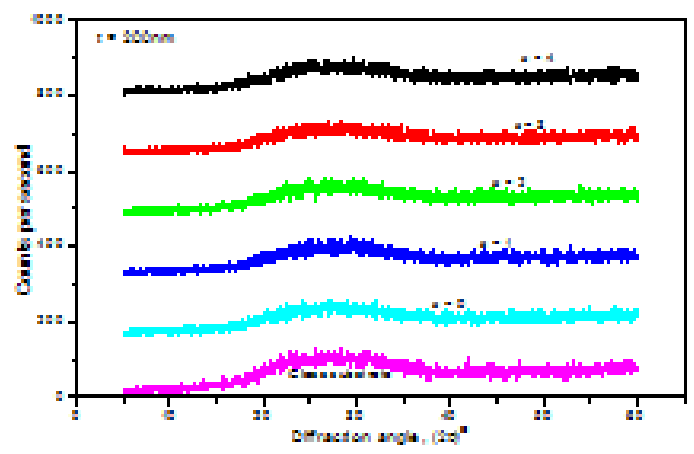

Figure 2: XRD patterns of $200 \mathrm{~nm} \mathrm{Ga}_{\mathrm{x}} \mathrm{Se}_{1-\mathrm{x}}$ thin films

\subsection{Transmittance and reflectance}

Fig. 3 (a \& b) shows transmittance and reflectance against wavelength for the as-deposited thin films. Transmittance is almost zero $(\sim 2 \%)$ at $500 \mathrm{~nm}$ due to maximum absorption of electromagnetic radiation. High reflectance $(\sim 30 \%)$ observed at the same wavelength results from low transmittance. Both the transmittance and reflectance spectra are characterized by interference patterns. This means that the as-deposited thin films of amorphous $\mathrm{Ga}_{x} \mathrm{Se}_{1-\mathrm{x}}$ are smooth and homogeneous. It is also observed that the transmittance in the visible region decreases with increase in gallium content. This is an indication of increase in the absorption of light with gallium content $[6,9]$. Further, the transmittance spectra shifts towards the visible region and this could be an indication of photosensitive films [10].
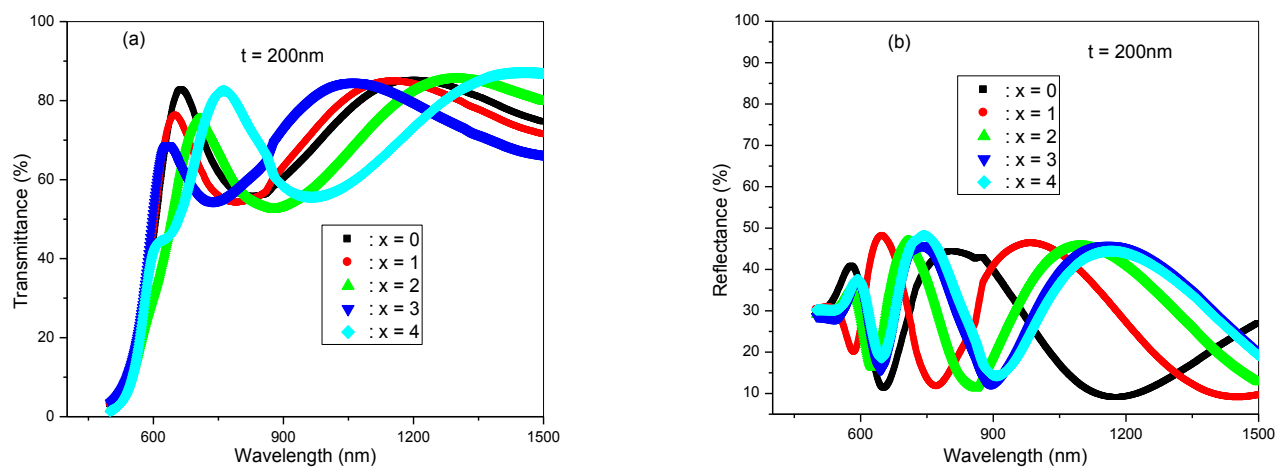

Figure 3: (a) Transmittance (\%) and (b) reflectance (\%) against wavelength (nm) for 200nm thin films 
From transmittance (\%) (Fig. 4a) and reflectance (\%) (Fig. 4b) against film thickness (nm), it is observed that the transmittance decreases with increase in film thickness and reflectance increases slightly. This is an indication of less transparent films with increase in film thickness. It also implies that the absorption coefficient increases with increase in film thickness. The increase in reflectance with film thickness correlates well with reduced transmittance.
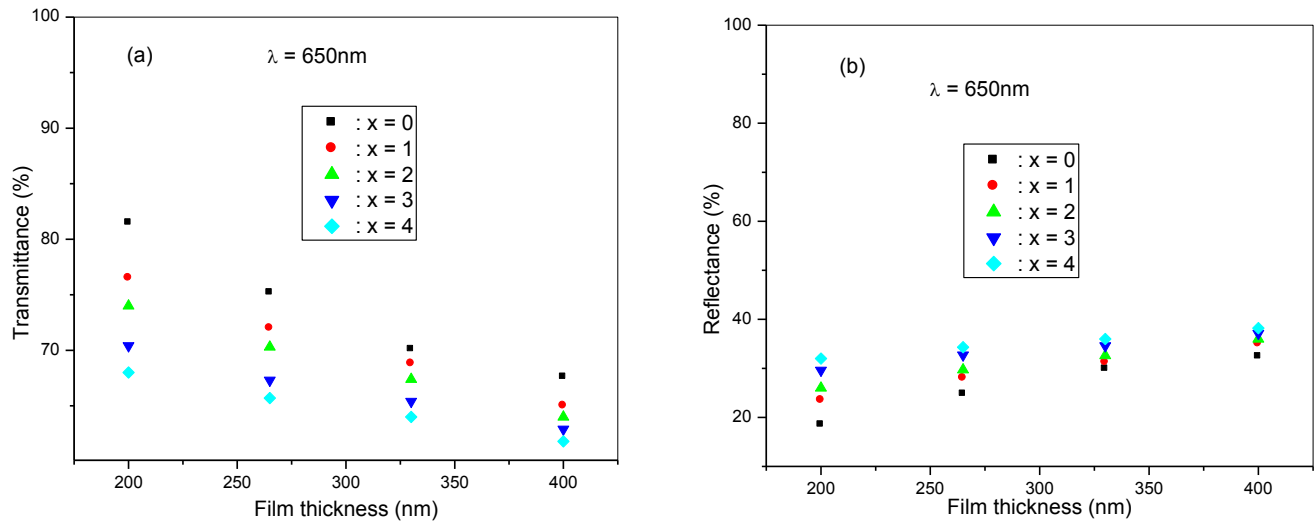

Figure 4: (a) Transmittance (\%) and (b) reflectance (\%) against film thickness (nm) at different gallium concentration and at $\lambda=650 \mathrm{~nm}$

\subsection{Optical parameters of as-deposited $\mathrm{a}-\mathrm{Ga}_{\mathrm{x}} \mathrm{Se} \mathrm{e}_{1-\mathrm{x}}$ thin films}

Absorption coefficient, extinction coefficient, refractive index, and real part of dielectric constant against wavelength $(\mathrm{nm})$ are shown in Fig. 5. At low wavelengths $(\leq 600 \mathrm{~nm})$, the absorption coefficient is high due to strong absorption of electromagnetic radiation in this region (Fig. 5a). Generally, the absorption coefficient decreases with increase in wavelength. The decrease in extinction coefficient with wavelength is due to the effect of absorption coefficient. The sinusoidal patterns observed in the region of high wavelength $(\geq$ $600 \mathrm{~nm}$ ) may be due to absorption in the as-deposited thin films (Fig. 5a \& b) [11].
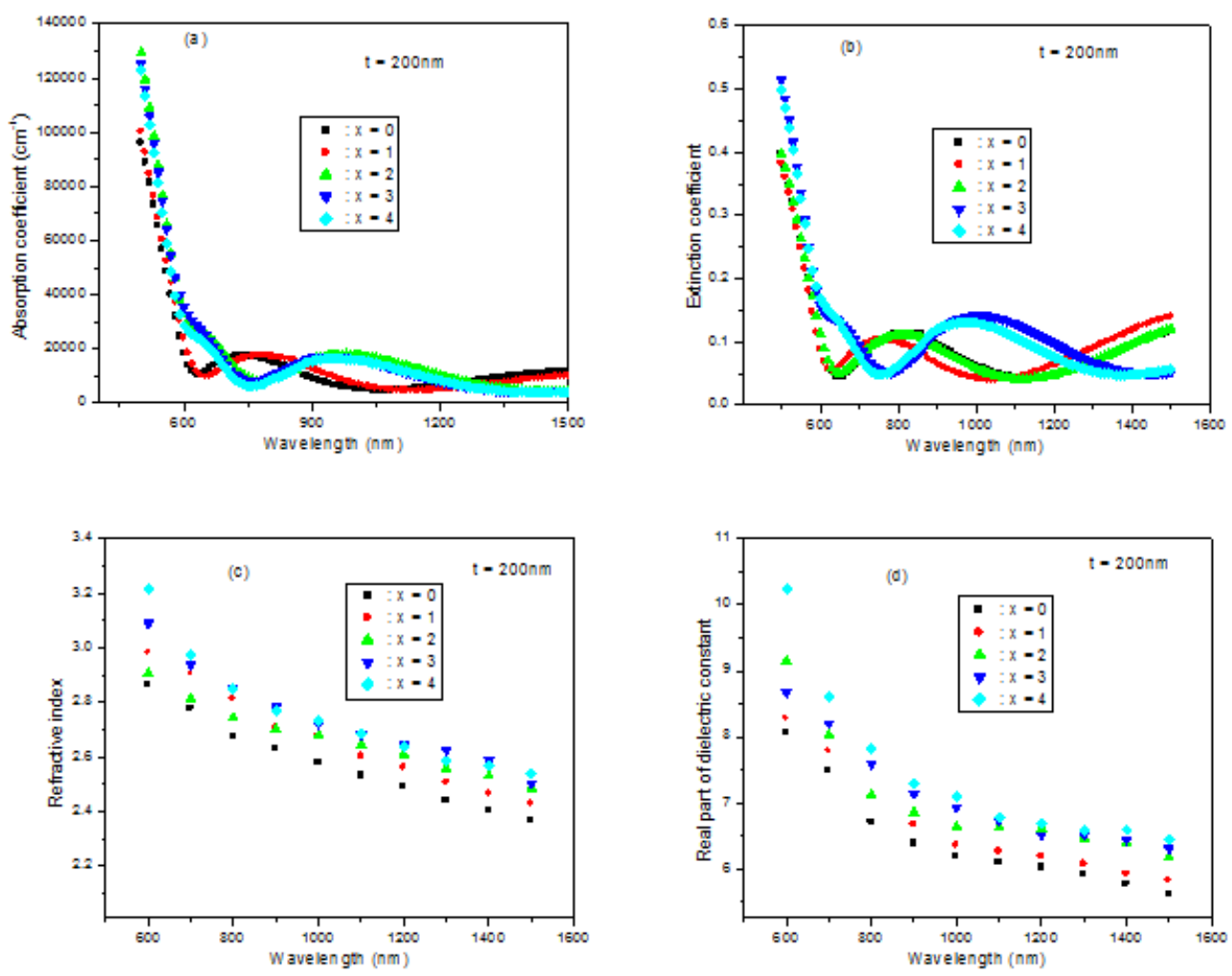

Figure 5: (a) Absorption coefficient, (b) exticntion coefficient, (c) refractive index, and (d) real part of dielectric constant against wavelength $(\mathrm{nm})$ for $200 \mathrm{~nm}$ thin films at different gallium concentrations 
The refractive index decreases with increase in wavelength ( $\mathrm{nm}$ ) (Fig. 5c). The decrease in refractive index with wavelength could be attributed to the strong effects of surface and volume imperfections in chalcogenide glasses [12]. It also indicates the normal dispersion in thin films. The refractive index (n) was calculated using the Swanepoel envelope method based on the equation (1) [13]:

$$
n=\left[N+\left(N^{2}-s^{2}\right)^{1 / 2}\right]^{1 / 2}
$$

where $\quad N=2 s \frac{T_{M}-T_{m}}{T_{M} T_{m}}+\frac{s^{2}+1}{2}, s$ is the refractive index of the glass substrate, $T_{M}$ and $T_{m}$ are the maximum and minimum transmittances [14]:

The real part and imaginary part of dielectric constant are given by equations (2) and (3), respectively

$$
\begin{gathered}
\varepsilon_{1}=n^{2}-k^{2} \\
\varepsilon_{2}=2 n k
\end{gathered}
$$

where $k$ is the extinction coefficient

Fig. 6 shows (a) absorption coefficient, (b) extinction coefficient, (c) refractive index, and (d) real part of dielectric constant against film thickness (nm) at different gallium content.
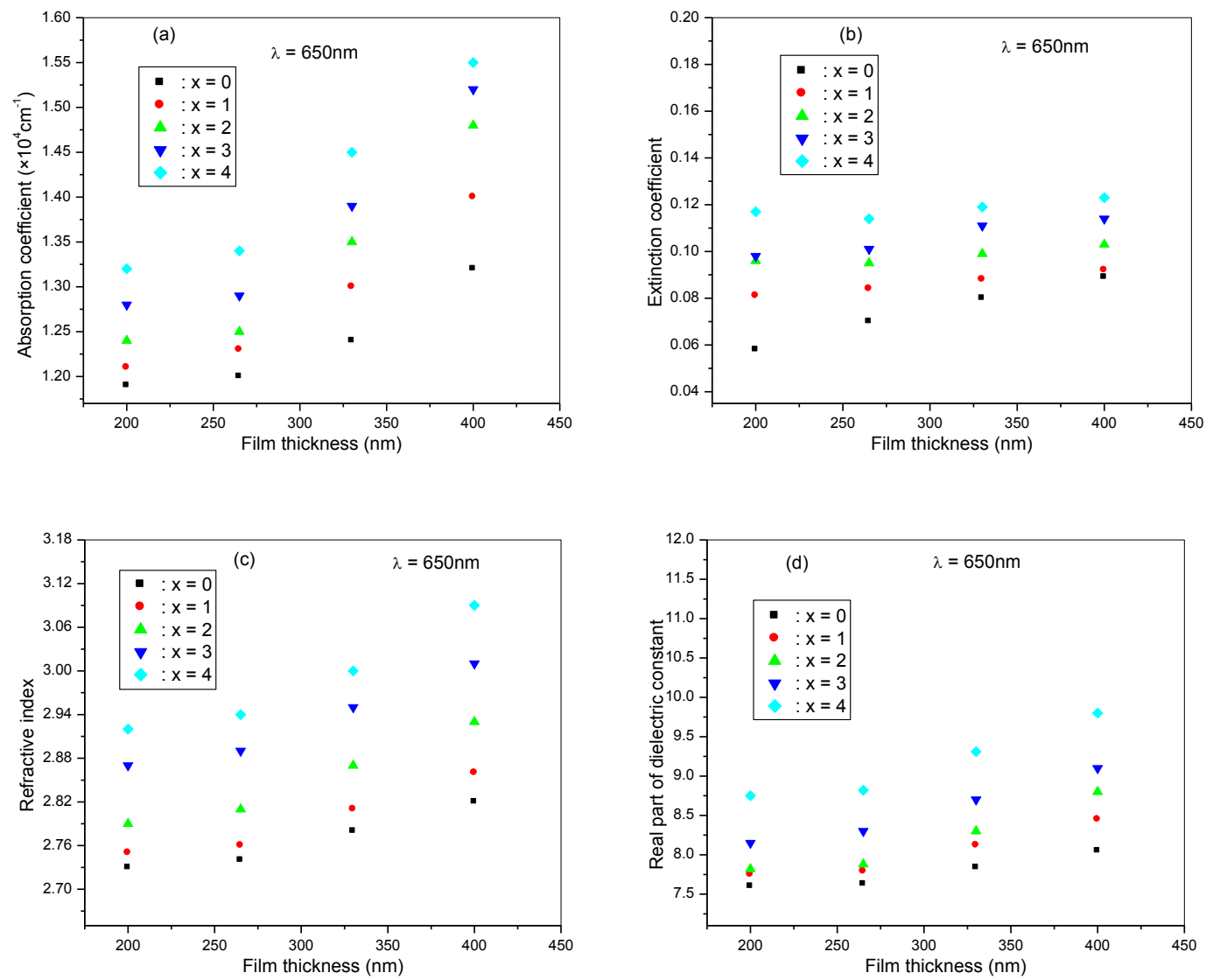

Figure 6: (a) Absorption coefficient, (b) extinction coefficient, (c) refractive index, and (d) real part of dielectric constant against film thickness $(\mathrm{nm})$ at different gallium content and at $\lambda=650 \mathrm{~nm}$

A close examination of Fig. 6(a) reveals that the thick films have higher absorption coefficient values than the thin films. This effect may be explained by the fact that thicker films increases the absorption path length of the deposited thin films decreasing the transmittance. The absorption coefficient is observed to increase with increase in gallium content. This may be an indication of an increase in photosensitivity of the deposited thin films with gallium addition. It is observed that there is an increase in extinction coefficient with increase in both the film thickness and gallium content (Fig. 6b) due to the increased absorption coefficient as already shown in Fig. 6(a) $[1,4,6]$. The refractive index of the deposited thin films generally increases with increase in both the film thickness and gallium content (Fig. 6c). Increasing the thickness and gallium content 
may have increased density of the deposited films leading to increased refractive index [15]. Increase in the real part of dielectric constant with gallium concentration and film thickness of the deposited thin films is observed in Fig. 6 (d) mainly due to the effect of the refractive index. This is as a result of the direct proportionality between the real part of dielectric constant and the refractive index and that the extinction coefficient is a small quantity.

Increase in imaginary part of dielectric constant with gallium concentration and film thickness of the deposited thin films is observed in Fig.7. This is due to the effect of the extinction coefficient since the imaginary part of dielectric constant is directly proportional to the extinction coefficient as can be observed from equation (3).

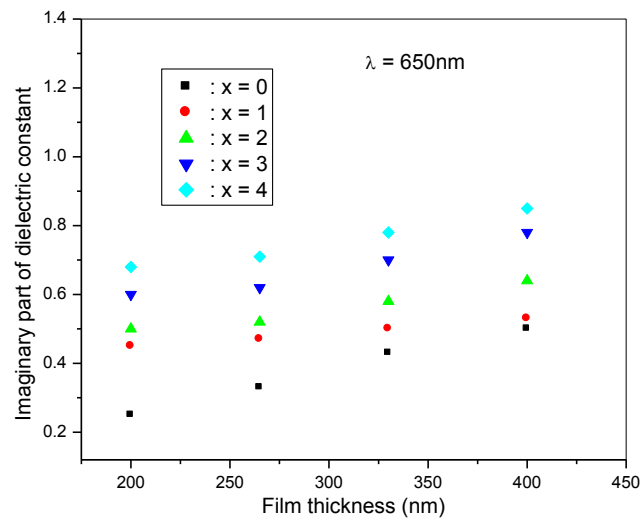

Figure 7: Imaginary part of dielectric constant against film thickness $(\mathrm{nm})$ at $\lambda=650 \mathrm{~nm}$

The band gap energy of a semiconductor is the minimum energy needed to move an electron from its bond state within an atom to a free state. This Free State is where the electron can be involved in conduction process. It is the energy difference between the conduction band and the valence band of the semiconductor material. To obtain the optical band gap energy $\left(\mathrm{E}_{\mathrm{g}}\right)$ of the deposited $\mathrm{a}-\mathrm{Ga}_{\mathrm{x}} \mathrm{Se}_{1-\mathrm{x}}(\mathrm{x}=0,1,2,3$, and 4 at. \%) thin films from Fig. 8(a), equation (4), according to Tauc [16] was used:

$$
(\alpha h v)^{1 / 2}=\left(h v-E_{g}\right)
$$

where $\alpha$ is the absorption coefficient, $h$ is Plank's constant and $v$ is the frequency
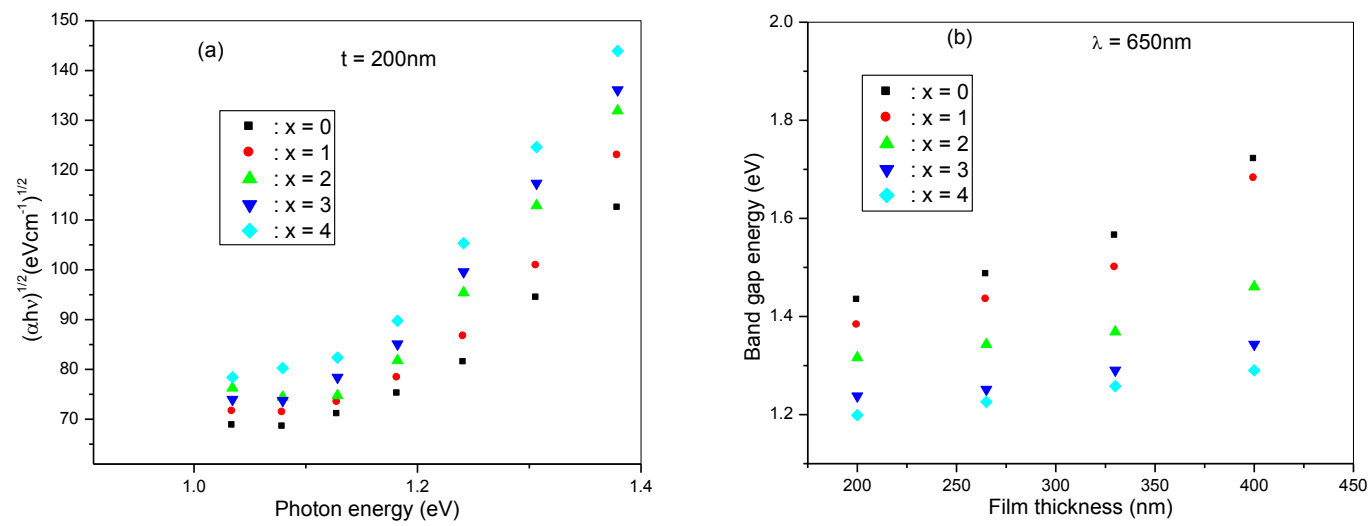

Figure 8: (a) $\quad(\alpha h v)^{1 / 2}\left(\mathrm{eVcm}^{-1}\right)^{1 / 2}$ against photon energy (eV) at 200nm film thickness and (b) Band gap energy (eV) against film thickness $(\mathrm{nm})$ at $\lambda=650 \mathrm{~nm}$

The band gap energy was found by extrapolating the graphs of Fig. 8 (a) to the energy axis. Other researchers have also found selenium rich binary and ternary glasses to obey the indirect rule transition $[1,4,6$, 9]. The optical band gap energy from Fig. 8(b) decreased with gallium addition. Similar trend has been observed by [6]. The decrease in the optical band gap energy may be due to decrease in cohesive energy of the system [17]. The band gap energy of the deposited $\mathrm{Ga}_{x} \mathrm{Se}_{1-\mathrm{x}}$ thin films varies with film thickness due to high density of dislocations resulting from gallium impurity in the system. Since dislocation density increases with increase in film thickness, it is expected that high dislocation density leads to increase in band gap energy as the film thickness increases. Greater deposition generally builds up more homogenous network which minimizes 
the number of defects and the localized states thereby increasing the electronic band gap energy [18]. The range of band gap energy for various film thicknesses are; $1.200 \mathrm{eV}-1.421 \mathrm{eV}(200 \mathrm{~nm}), 1.205 \mathrm{eV}-1.507 \mathrm{eV}(265 \mathrm{~nm})$, $1.211 \mathrm{eV}-1.564 \mathrm{eV}(330 \mathrm{~nm})$, and $1.234 \mathrm{eV}-1.712 \mathrm{eV}(400 \mathrm{~nm})$. A film thickness of $265 \mathrm{~nm}$ yields best range for the band gap energy that can be useful in the production of thin film solar cells.

\section{Conclusion}

Thin films of amorphous $\mathrm{Ga}_{\mathrm{x}} \mathrm{Se}_{1-\mathrm{x}}(\mathrm{x}=0,1,2,3$, and 4 at. \%) have shown to be photosensitive with respect to gallium content and film thickness. This is due to the shift of transmittance spectra towards the visible region. As a result, thin films of Ga-Se alloy can be used to produce thin film solar cells and other photovoltaic devices. Since band gap energy increases with increase in film thickness, a film thickness of $265 \mathrm{~nm}$ based on this study can yield optimized energy gap for a solar cell based on a- $\mathrm{Ga}_{x} \mathrm{Se}_{1-\mathrm{x}}$ thin films.

\section{Acknowledgements}

The authors wish to thank Mr. Boniface Muthoka and Ms. Everlyne Odera for their technical assistance during the experimental stage.

\section{References}

[1] N. Mohd and M. Zulfequar, DC Conductivity and dielectric behaviour of glassy $\mathrm{Se}_{100-\mathrm{x}} \mathrm{Zn}_{\mathrm{x}}$ alloy, Journal of Inorganic NonMetallic Materials, 2, 2012, 11-17.

[2] M. Popescu, Self-organization in amorphous semiconductors and chalcogenide glasses, Journal of Advanced Materials, 8(6), 2006, 2164-2168.

[3] E. A. Davis, Electronic and structural properties of amorphous semiconductors (Academic Press: London, 1973).

[4] A. A. Mulama, J. M. Mwabora, A. O. Oduor, and C. M. Muiva, Optical properties and Raman studies of amorphous Se-Bi thin films, The African Review of Physics, 9(6), 2014, 33-37.

[5] P. K. Johnson, The effect of trapping defects on CIGS solar-cell performance, doctoral diss., Colorado State University, USA, 2003.

[6] F. I. Mustafa, S. Gupta, N. Goyal, and S. K. Tripathi, Temperature dependence of the energy gap, refractive index and opticaloscillator parameters of amorphous $\mathrm{Ga}_{\mathrm{x}} \mathrm{Se}_{1-\mathrm{x}}(\mathrm{x}=0.4,0.5,0.6)$ thin films, Physica Status Solidi C, 6(1), 2009, 135-138.

[7] M. A. Majeed Khan, S. Kumar, M. Husain, and M. Zulfequar, Thermal properties of Selenium-Bismuth glassy alloys, Chalcogenide Letters, 4(12), 2007, 147-153.

[8] R. Chauhan, A. Tripathi, A. K. Srivastava, and K. K. Srivastava, Effect of swift heavy ion irradiation on optical and structural properties of amorphous Ge-As-Se thin films, Chalcogenide Letters, 10(2), 2013, 63-71.

[9] M. A. Majeed Khan, M. Zulfequar, and M. Husain, Optical investigation of a-Se $\mathrm{e}_{100-\mathrm{x}} \mathrm{Bi}$ x alloys, Optical Materials, $22,2003,21-29$.

[10] V. Pandey, S. K. Tripathi, and A. Kumar, Optical properties of amorphous $\mathrm{Se}_{100-\mathrm{x}} \mathrm{Sb}_{\mathrm{x}}$ thin films, Journal of Ovonic Research, 2(4), 2006, 67-76.

[11] K. Kumar, P. Sharma, S. C. Katyal, and N. Thakur, Optical parameters of ternary Te ${ }_{15}\left(\operatorname{Se}_{100-\mathrm{x}} \mathrm{Bi}_{\mathrm{x}}\right)_{85}$ thin films deposited by thermal evaporation, Phys. Scr., 84(045703), 2011, 1-6.

[12] G. A. N. Connell, Optical properties of amorphous semiconductors (Springer Berlin: Heidelberg, 1985).

[13] R. Swanepoel, Determination of the thickness and optical constants of amorphous silicon, Journal of Physics E: Science Instrum, $16,1983,1214-1218$.

[14] A. Goswami, Thin film fundamentals (New Age International Publishers: Delhi, India, 1997).

[15] C. T. Wee, Optical properties of amorphous selenium films, M. Sc. diss., University of Saskatchewan, Saskatoon, Canada, 2006.

[16] J. Tauc, Amorphous and liquid semiconductors (Plenum Press: London, 1974).

[17] C. M. Muiva, T. S. Sathiaraj, and J. M. Mwabora, Chemical bond approach to optical properties of some flash evaporated $\mathrm{Se}_{100-\mathrm{x}} \mathrm{Sb}_{\mathrm{x}}$ chalcogenide alloys, European Physical Journal of Applied Physics, 59(10301), 2012, 1-7.

[18] E. A. Davis and N. F. Mott, Electronic processes in non-crystalline materials (Clarendon Press: Oxford, 1979). 